EISSN: 2706 -7947 ISSN: 2077- 4613

DOI: $10.36632 / \mathrm{mejas} / \mathbf{2 0 2 1 . 1 1 . 2 . 4 5}$

Journal homepage: www.curresweb.com

Pages: 579-588

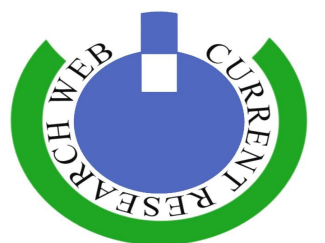

\title{
Cardiac Protective Effect of Terminalia muelleri Extract in Rat Model of Diabetes Mellitus
}

\section{Sawsan Ahmed Nasr ${ }^{1}$ and Mona Saber Hamed ${ }^{2}$}

${ }^{1}$ Physiology Department and ${ }^{2}$ Biochemistry Department, Egyptian Drug Authority, Formerly Known As National Organization for Drug Control and Research, Egypt.

Received: 15 April $2021 \quad$ Accepted: 30 May $2021 \quad$ Published: 25 June 2021

\begin{abstract}
Cardiovascular disease is the major cause of morbidity and mortality in people with type 2 diabetes. The present study aims to evaluate the cardiac protective effect of Terminalia muelleri (TM) in diabetic rats. Treatment of diabetic rats with a daily oral dose of (TM) extract ( $200 \mathrm{mg} / \mathrm{kg} \mathrm{b}$.wt) or pioglitazone tablet $(1.58 \mathrm{mg} / \mathrm{kg}$ b.wt) for four weeks resulted in a significant decrease in the glucose, total cholesterol (TC), triglycride (TG), low density lipoprotein cholesterol (LDL-C), malondialdehyde (MDA), aspartate amino transferase (AST) and creatin kinase-MB (CK-MB) activities as well as homocysteine (Hcy) and troponin-1 (cTn-1) levels in association with a significant increase in high density lipoprotein cholesterol (HDL-C) level, glutathione (GSH) content and nuclear factor-erythroid factor 2-related factor $2(\mathrm{Nrf} 2)$ level when compared with diabetic rats. These data suggest that TM might be an effective antidiabetic agent in the prevention of diabetic complication. TM extract could help in combating against oxidation damage in cardiac tissue.
\end{abstract}

Keywords: Hyperglycemia, Hyperlipidemia, Oxidative stress, Homocysteine, Troponin-1 and Terminalia muelleri

\section{Introduction}

Diabetes mellitus (DM) is a group of metabolic disorder disease that characterized by hyperglycemia resulting from defects in insulin secretion or action or both, that affects carbohydrate, lipid, protein and nucleic acid metabolism (Al-Lawati, 2017 and Poznyak et al., 2020). Different factors have been related to the higher production of free radicals in diabetes which acts by activating oxidative stress related pathways (Asmat et al., 2016). Oxidative stress plays a central role in the onset of diabetes mellitus as well as in the development of vascular complications of the disease (Pitocco et al., 2013).

In addition, to the impact of oxidative stress in the development of cardiovascular disease in diabetes, hyperhomocysteinemia is one of the suggested mechanisms that explains the relationship between the increased level of homocysteine (Hcy) and cardiovascular disease, while it has been hypothesized that, the vascular lesions resulting from hyperhomocysteinemia could be involved in cardiovascular disease that is the major cause of morbidity and mortality in people with type 2 diabetes (McCully, 2017; Einarson et al., 2018).

Herbal medicines have recently attracted much attention as alternative medicines as they are useful for treating or preventing life style related disorders (Parkash et al., 2018). Hypoglycemic herbs are widely used as non-prescription treatment for diabetes. However, few herbal medicines have been well characterized and demonstrated the efficacy in systematic clinical trials (Mahmood et al., 2011). Members of genus Terminalia are widely used in traditional medicine for the treatment of various ailments. Many pharmacological activities were reported for different species of Terminalia including antioxidant, hepatoprotective, antihyperlipidemic, antidiabetic, anti- inflammatory and cytotoxic activities. Terminalia muelleri Benth, exerts hepatoprotective, nephroprotective and antioxidant activity (Fahmy et al., 2016). Terminalia muelleri is a promising medicinal plant as it exhibited potent

Corresponding Author: Mona Saber Hamed, Biochemistry Department, Egyptian Drug Authority, Formerly Known As National Organization for Drug Control and Research, Egypt. E-mail: monasaber1282011@yahoo.com 
antioxidant scavenging activity (El-Kashak et al., 2017). Therefore, the present study will highlight the cardiac protective effect of Terminalia muelleri in diabetic rats. Pioglitazone was used in the current study as a reference hypoglycemic drug.

\section{Materials and Methods}

\subsection{The preparation of Terminalia muelleri extract}

The ethanolic extract of Terminalia muelleri leaves were prepared according to the method described by Fahmy et al., (2016). Terminalia muelleri leaves were collected from Zoo botanical garden, Giza city, Egypt. Leaves were washed and air-dried. Dried leaves were grounded into powder using electric blinder. Two kilogram of $T$. muelleri leaves powder was extracted five times with $85 \%$ ethanol. The total extract was concentrated and lyophilized to obtain about $400 \mathrm{~g}$ powder.

\subsection{Animals and experimental design}

A total number of 40 male albino rats $(180-200 \mathrm{~g})$ from the laboratory stock colony of National Organization for Drug Control and Research (NODCAR) were used in the present study. The animals were kept under normal environmental conditions for two weeks before the initiation of the experiment. The animals were allowed free access to water and fed on a standard diet. This study was approved by the local ethics committee of NODCAR. The approval number is NODCAR/II/3/2020.

\subsection{Experimental design}

\subsubsection{Induction of Diabetes}

Diabetes was induced in $12 \mathrm{~h}$ fasted rats by injecting freshly prepared streptozotocin (STZ) subcutaneously with an initial dose of $27.5 \mathrm{mg} / \mathrm{kg}$ b.wt, booster injection of three successive doses $(11.25 \mathrm{mg} / \mathrm{kg}$ b.wt) were given within four weeks according to Said et al., (2000). Rats confirmed with permanent glucose level above $200 \mathrm{mg} / \mathrm{dL}$ were considered to be diabetic and used for the experiment.

The animals were divided into five groups ( $\mathrm{n}=8$ per group):

Group (1): Normal control which received a regular diet through the experimental period.

Group (2): Positive control rats (STZ).

Group (3): Rats treated with a daily oral dose of Terminalia muelleri extract (200 mg/kg b.wt) for four weeks.

Group (4): Diabetic rats treated with a daily oral dose of Terminalia muelleri extract $(200 \mathrm{mg} / \mathrm{kg}$ b.wt) for four weeks (Fahmy et al., 2016).

Group (5): Diabetic rats treated with a daily oral dose of pioglitazone (1.58mg $/ \mathrm{kg} \mathrm{b.wt)}$ for four weeks (Paget and Barnes 1964).

\subsubsection{Blood sampling}

At the end of the experimental period, blood samples were collected from the retro-orbital plexus in heparinized and non-heparinized tubes, plasma and sera were separated. The blood samples were centrifuged at $4000 \mathrm{rpm}$ for 15 minutes at cooling centrifuge to separate plasma, which is used for the determination the levels of glucose, total cholesterol (TC), triglycerides (TG), high density lipoprotein cholesterol (HDL-C) and aspartate amino transferase (AST) activity. Separated serum was used to measure homocysteine (Hcy), troponin-1 (cTn-1) levels and creatine kinase-MB (CK-MB) activity. Fresh heart tissue were removed from the decapitated rats for the determination of reduced glutathione (GSH), malondialdehyde (MDA) and nuclear factor-erythroid 2-related factor 2 (Nrf2).

\subsubsection{Biochemical analysis}

Glucose was determined according to Trinder (1969) method. TC was determined colorimetricaly according to the method of Richmond (1973). HDL-C was determined according to Warnick and Albers (1978) method. TG was determined according to Fassati and Prencipe (1982) method. Low density lipoprotein cholesterol (LDL-C) was calculated according to the formula of Friedewald et al., (1972) as following: 


$$
\mathrm{LDL}-\mathrm{C}=T C-\left(\frac{T G}{5}-H D L-C\right)
$$

AST activity was determined by kinetic method (Bergmeyer et al., 1986). CK-MB according to the method of Abbot et al., (1984). Enzyme-linked immunosorbent (ELISA) tecnique was used for the determination of serum Hcy according to Shipchandler and Moore (1995), serum cTnI level as described by Dean (1998) using kits provided by Jena Bioscience (Germany) and Nrf2 in heart tissue according to the method of Kubben et al., (2016). GSH content in heart tissue was determined according to Beutler et al., (1963) method, estimation of MDA according to the method of Buege and Aust (1978).

\subsection{Statistical Analysis}

Data are presented as mean values $\pm \operatorname{SE}(n=6)$. Differences between groups were assessed by one-way analysis of variance (ANOVA). Subsequent multiple comparisons between the different groups were analyzed by Duncan's multiple comparisons test. Data were statistically analyzed using the statistical package for social science (SPSS 16.0 software) values at $p<0.05$ were considered significant (Dawson and Trapp, 2004).

\section{Results}

\subsection{TM extract ameliorates STZ-induced hyperglycemia:}

Table (1) shows that, treatment of diabetic rats with TM extract or pioglitazone tablet for four weeks caused a significant decrease in the glucose level when compared with STZ- treated group.

Table 1: Effect of the oral administration of TM extract or pioglitazone tablet for four weeks on blood glucose levels in normal and diabetic rats

\begin{tabular}{ll}
\hline Groups & Gloucose $(\mathbf{m g} / \mathbf{d L})$ \\
\hline Control & $116.33^{\mathrm{a}} \pm 2.319$ \\
STZ & $237.33^{\mathrm{d}} \pm 3.456$ \\
TM Extract & $113.00^{\mathrm{a}} \pm 2.600$ \\
TM Extract + STZ & $153.66^{\mathrm{c}} \pm 3.572$ \\
Drug* + STZ & $144.66^{\mathrm{b}} \pm 2.347$ \\
\hline
\end{tabular}

Values are mean \pm SE $(n=6)$.

The presence of different letters means significant differences between groups. ANOVA test followed by Duncan's multiple comparisons between groups at $\mathrm{P}<0.05$ were employed.

* Pioglitazone

\subsection{TM extract restores lipid profile in diabetic rats}

Data presented in Table (2) reveals that, the induction of diabetes caused a disturbance in lipid profile as manifested by the significant increase in the levels of TC, LDL-C and TG in association with a marked reduction of HDL-C level in STZ-induced diabetic rats. Meanwhile, the oral administration of TM, or the administration of the drug to diabetic rats caused a significant decrease in TC, LDL-C and TG levels in association with a significant improvement in HDL-C level.

Table 2: The administration effects of TM extract or pioglitazone tablet for four weeks on the plasma levels of total cholesterol, HDL-cholesterol, triglyceride and LDL-cholesterol in normal and diabetic rats

\begin{tabular}{lllll}
\hline Groups & Cholesterol (mg/dL) & HDL-C (mg/dL) & Triglyceride (mg/dL) & LDL-C \\
\hline Control & $76.95 \pm 1.96^{\mathrm{a}}$ & $27.37 \pm 0.904^{\mathrm{c}}$ & $144.58 \pm 6.17^{\mathrm{a}}$ & $12.56^{\mathrm{a}} \pm 0.348$ \\
STZ & $110.66 \pm 4.66^{\mathrm{c}}$ & $15.33 \pm 0.42^{\mathrm{a}}$ & $200.66 \pm 1.20^{\mathrm{c}}$ & $53.00^{\mathrm{c}} \pm 1.80$ \\
Extract & $69.33 \pm 2.52^{\mathrm{a}}$ & $25.0 \pm 1.23^{\mathrm{c}}$ & $139.83 \pm 3.07^{\mathrm{a}}$ & $13.68^{\mathrm{a}} \pm 0.473$ \\
Extract+ STZ & $84.16 \pm 3.02^{\mathrm{b}}$ & $19.66 \pm 1.35^{\mathrm{b}}$ & $179.33 \pm 1.11^{\mathrm{b}}$ & $30.158^{\mathrm{b}} \pm 1.167$ \\
${ }^{* D r u g}+\mathbf{S T Z}$ & $83.16 \pm 1.70^{\mathrm{b}}$ & $19.16 \pm 0.60^{\mathrm{b}}$ & $177.01 \pm 2.74^{\mathrm{b}}$ & $28.30^{\mathrm{b}} \pm 1.149$ \\
\hline
\end{tabular}

Values are mean $\pm \mathrm{SE}(\mathrm{n}=6)$.

In the same column, the presence of different letters means significant differences between groups. ANOVA test followed by Duncan's multiple comparisons between groups at $\mathrm{p}<0.05$ were employed.

*Pioglitazone 


\subsection{TM extract attenuates the oxidative heart injury in diabetic rats:}

The obtained data in Table (3) revealed that, the subcutaneous injection of STZ exerted a significant decline in cardiac GSH content when compared with control non-treated group. Meanwhile, MDA recorded a significant increase in STZ-treated rats. It is also recorded that the administration of TM or the reference drug to diabetic rats caused a significant reduction in the level of MDA and a significant increase in GSH content with respect to STZ-treated group.

Table 3: Effect of the oral administration of TM extract or pioglitazone tablet for four weeks on reduced GSH content and MDA level on the cardiac tissue in normal and diabetic rats

\begin{tabular}{lll}
\hline Groups & GSH $(\boldsymbol{\mu M} / \mathbf{g}$ tissue $)$ & MDA $(\mathbf{n M} / \mathbf{g}$ tissue) \\
\hline Control & $13.41 \pm 0.31^{\mathrm{b}}$ & $26.40 \pm 1.18^{\mathrm{a}}$ \\
STZ & $8.09 \pm 0.51^{\mathrm{a}}$ & $54.55 \pm 3.11^{\mathrm{d}}$ \\
Extract & $12.94 \pm 0.59^{\mathrm{b}}$ & $29.26 \pm 1.00^{\mathrm{ab}}$ \\
Extract + STZ & $12.35 \pm 2.08^{\mathrm{b}}$ & $39.00 \pm 1.25^{\mathrm{c}}$ \\
${ }^{\text {*Dug }}+$ STZ & $12.91 \pm 0.58^{\mathrm{b}}$ & $34.34 \pm 0.81^{\mathrm{bc}}$ \\
\hline
\end{tabular}

Values are mean $\pm \operatorname{SE}(n=6)$.

In the same column, the presence of different letters means significant differences between groups. ANOVA test followed by Duncan's multiple comparisons between groups at $\mathrm{p}<0.05$ were employ.

*Pioglitazone

Figure (1) shows that induction of diabetes causes a significant reduction in Nrf2 transcription factor while the oral administration of TM, or the drug to diabetic rats caused a significant increase in Nrf2 when compared with STZ group and TM is more effective than drug.

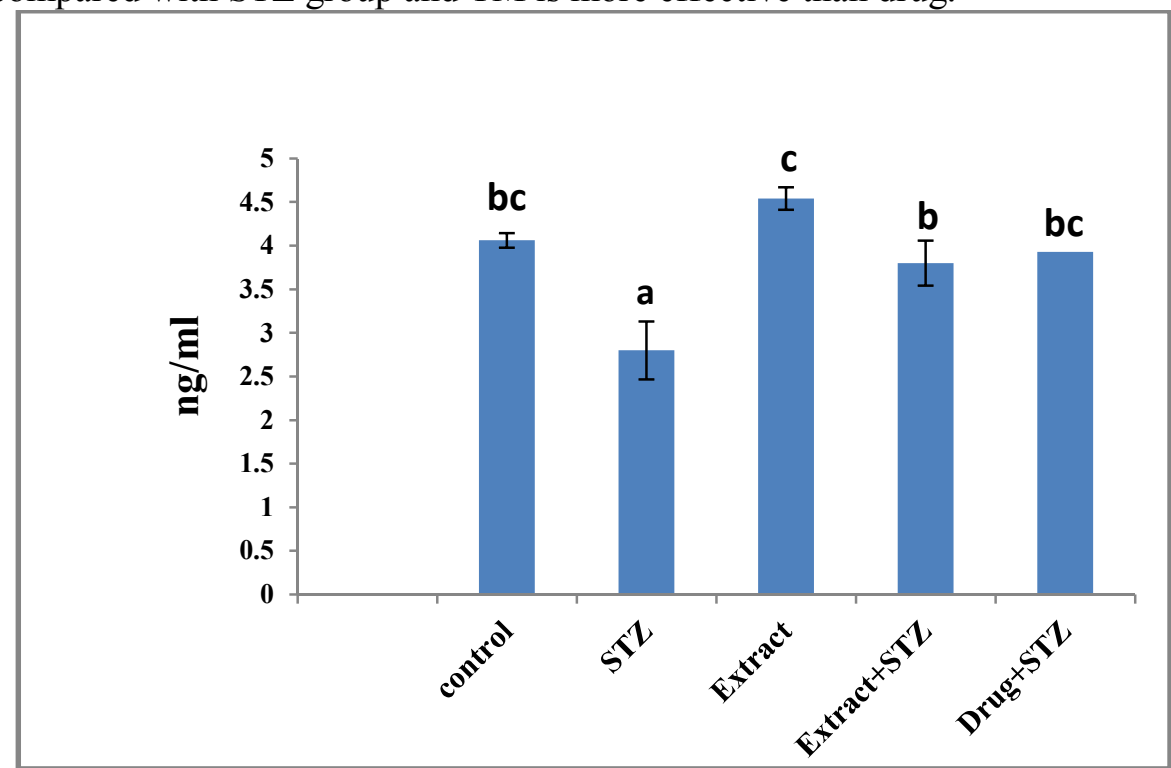

Fig 1: Effect of the oral administration of examined materials on cardiac tissue $\mathrm{Nrf} 2(\mathrm{ng} / \mathrm{ml})$ of normal and diabetic rats for four weeks

Each bar represents a mean value \pm SE $(n=6)$. The presence of different letters on each bar means significant differences between groups. ANOVA test followed by multiple comparisons between groups at $p<0.05$ were employed.

\subsection{TM extract ameliorates cardiac function in diabetic rats}

The subcutaneous injection of STZ exerted a significant increase in AST activity when compared with control non-treated group, the obtained results also revealed that, the administration of the examined materials to rats exerted a significant improving effect on AST activity to diabetic rats when compared with STZ-treated rats (Fig 2). 


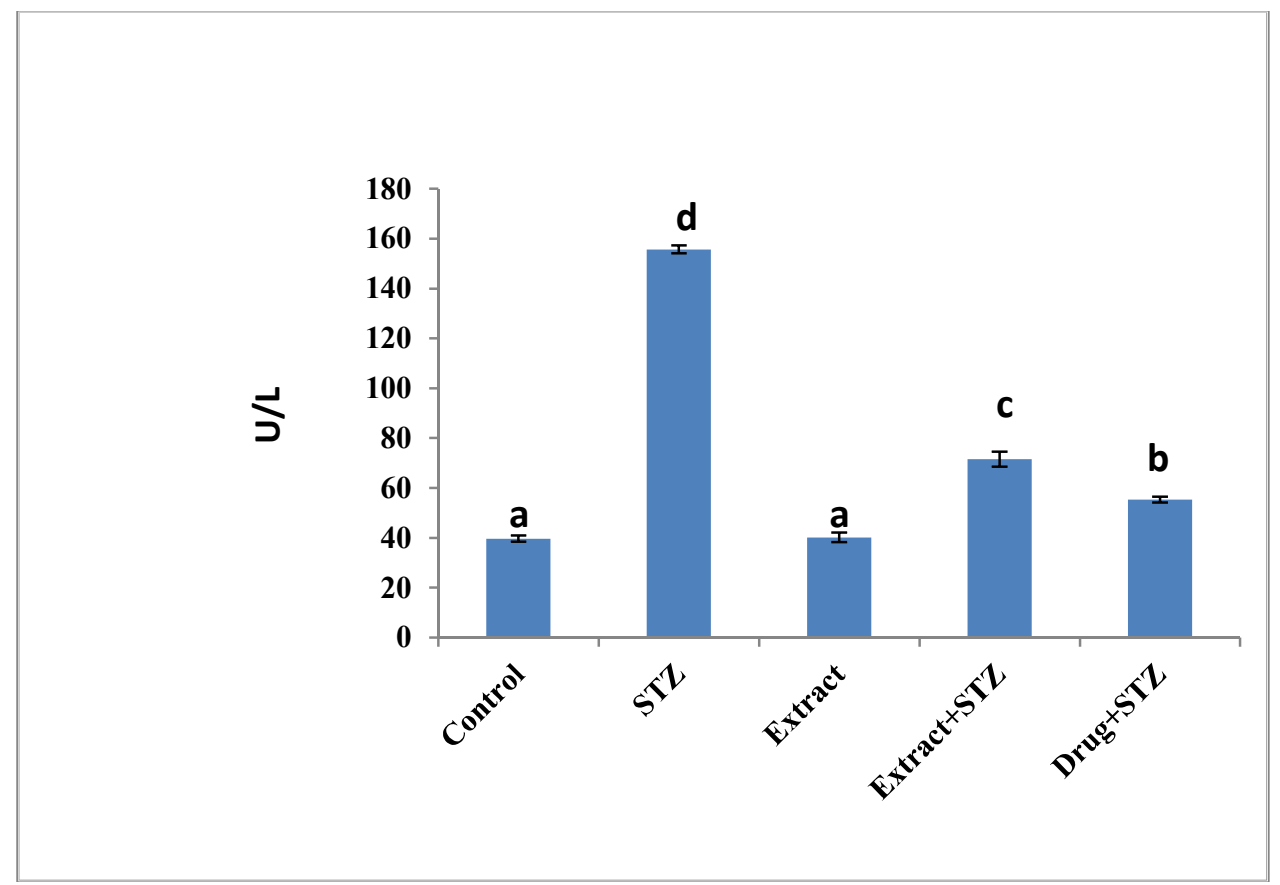

Fig 2: Effect of the oral administration of examined materials on AST activity (U/L) on normal and diabetic rats for four weeks

Each bar represents a mean value $\pm \mathrm{SE}(\mathrm{n}=6)$. The presence of different letters on each bar means significant differences between. ANOVA test followed by multiple comparisons between groups at $p<0.05$ were employed.

\subsection{TM extract alleviates the risk factor of hyperhomocysteinemia in diabetic rats}

The presented results in Table (4) reveal that, the treatment of normal rats with STZ induced a significant increases in the levels of Hcy, cTn-1 and the activity of CK-MB when compared with normal control group. Meanwhile, the oral administration of TM extract or pioglitazone tablet to diabetic rats caused a significant decrease in the level of Hcy, cTn-1 and CK-MB activity when compared with diabetic rats.

Table 4: Effect of the oral administration of TM extract or pioglitazone tablet for four weeks on the serum levels of homocysteine, troponin-1 and CK-MB activity in normal and diabetic rats

\begin{tabular}{llll}
\hline Groups & Homocysteine $(\mathbf{p m o l} / \mathbf{m l})$ & Troponin-1 (pg/ml) & CK-MB (U/L) \\
\hline Control & $82.5 \pm 2.06^{\mathrm{a}}$ & $111.42 \pm 4.28^{\mathrm{a}}$ & $118.10 \pm 5.34^{\mathrm{a}}$ \\
STZ & $251.83 \pm 7.35^{\mathrm{c}}$ & $237.83 \pm 1.58^{\mathrm{c}}$ & $334.66 \pm 14.37^{\mathrm{d}}$ \\
TM Extract & $84.25 \pm 3.41^{\mathrm{a}}$ & $111.17 \pm 4.1^{\mathrm{ab}}$ & $192.83 \pm 2.63^{\mathrm{a}}$ \\
TM Extract + STZ & $122.66 \pm 3.58^{\mathrm{b}}$ & $129.5 \pm 6.9^{\mathrm{b}}$ & $243.33 \pm 5.63^{\mathrm{b}}$ \\
${ }^{*}$ Drug + STZ & $124.83 \pm 3.18^{\mathrm{b}}$ & $119 \pm 2.7^{\mathrm{ab}}$ & $214.83 \pm 6.94^{\mathrm{ab}}$ \\
\hline
\end{tabular}

Values are mean $\pm \mathrm{SE}(\mathrm{n}=6)$.

In the same column, the presence of different letters means significant differences between groups. ANOVA test followed by Duncan's multiple comparisons between groups at $\mathrm{p}<0.05$ were employed. *Pioglitazone

\section{Discussion}

Diabetes mellitus (DM) represents a major public health problem and is also conciderd as a major cause of mortality. DM is an endocrine-metabolic disorder of multiple etiologies characterized by chronic hyperglycemia that leads via several mechanisms (glucose auto oxidation, stimulation of polyol pathway, activation of reduced form of nicotinamide adenine dinucleotide phosphate oxidase and production of advanced glycation end products) to increase production of reactive oxygen species (ROS). The resulting oxidative stress can play a key role in diabetes pathogenesis (Moussa 2008; Lee and Kang 2013). It has been shown that, oxidative stress starts at early onset of diabetes. The consequence of oxidative stress can be involved in the structural tissue damage and in the development of diabetic complications (Modak et al., 2007 and Matough et al., 2012). 
The incidence of hyperglycemia is confirmed in the current study by the well marked increase of glucose level. In the present study, the induction of diabetes caused a significant increase in plasma levels of TC, TG and LDL-C in association with a marked decrease in HDL-C level. These findngs clearly proved that the disturbance of lipid profile due to the induction of hyperlipidemia in diabetic rats. Data of this study are in agreement with the results of Rajeswari and Rajagopalan (2013) and Poznyak et al., (2020) who attributed this disturbance in lipid profile to the increased mobilization of free fatty acids (FFA) from adipose tissue in diabetic rats. Moreover, the obtained hypercholesterolemia in the current study is in consistent with SubashBabu et al., (2007) and Poznyak et al., (2020) who attributed the increase of cholesterol to the increased intestinal absorption and increased cholesterol biosynthesis in diabetic rats. In the current study, hypertriglyceridemia that indicated in diabetic rats could be attributed to increase in the activity of hormone-sensitive lipase, which catalysis the mobilization of fatty acids from triacylglycerols stored in adipocytes as described by Almeida et al., (2012). As shown in our results the decrease of plasma HDL-C level in diabetic rats is in consistent with the results of Nwaneri-Chidozie et al., (2014) who referred this decrease to the diminished lecithin cholesterol transferase activity. It is clearly proved that diabetes mellitus is associated with profound alteration in lipid profile that may be a risk factor for coronary heart disease.

Lipid peroxidative end product MDA serves as a main biomarker to assess the level of lipoperoxidative tissue damage (Khan and Ahmed, 2009). The obtained significant increase in MDA and the significant decrease in the content of GSH in heart tissue proved that, the pathogenesis of DM is predominantly related to the over generation of ROS as well as impaired antioxidant ability. This disturbance in the prooxidants/antioxidants balance was further verified by the low level of Nrf2 transcription in heart tissue. The transcription factor nuclear factor-erythroid $2 \mathrm{p} 45$-related factor 2 (Nrf2, also called NFE2L2) is a master regulator of cellular redox status and detoxification response (Chen et al., 2018 and Zang et al., 2020). It plays a vital role in maintaining the oxidative homeostasis by regulating multiple downstream antioxidants. Significantly reduced Nrf2 expression has been observed in the heart of diabetic rat's model. These findings suggest adaptive over expression of Nrf2 to combat early oxidative damage in diabetes, which is overcome by sustained ROS production and exhaustion of the antioxidant defenses (Hu et al., 2018 and Wang et al., 2018 ).

Administration of STZ to rats produced leakage associated with a significant elevation in the serum levels of cardiac biomarker CK-MB and AST. CK-MB is an isoenzyme of CK and a serum marker of myocardial injury. CK-MB activity was reported to increase in diabetic rats, and may serve as a marker for cardiovascular risk and cardiac muscular damage (Wang et al., 2015). Troponin-1 (cTn-1) is considerd as one of the main components of contractile apparatus in cardiac and skeletal muscle. Also it is a protein released from the heart cells when they are damaged. It is only found in the heart muscle, making it useful in diagnosing damage to the heart muscle (Wu et al., 2017). In the present study, we found a well marked increase in the serum levels of cTn- 1 in diabetic rats. These findigs proved the incidence of cardiac insults in diabetic rats.

Homocystein (Hcy) is a homologue of the amino acid cysteine, differing by an additional methylene (-CH2-) group. It is biosynthesized from methionine by the removal of its terminal methyl group and can be recycled into methionine or converted into cysteine with the aid of B-vitamins (Shaikh et al., 2012). There has been an indication towards a significant correlation between hyperhomocysteinemia and cardiovascular disease and its complications such as heart attacks and strokes (Belcastro and Striano 2012 ; Baszczuk and Kopczynski 2014). In the present study, induction of diabetes caused a significant increase in the level of total homocysteine (tHcy) in serum.

The elevation of Hcy level has both atherogenic and thrombogenic effects also causes endothelial dysfunction by enhancing oxidative stress. High serum Hcy levels may cause thrombogenic activity by altering coagulation system and stimulate platelet aggregation. Excess of serum Hcy level seemed to be related with enhanced lipid peroxidation which also predisposes to atherosclerosis (Bansal et al., 2016). In this way, vascular lesion resulting from hyperhomocysteinemia, could be involved lesion of endothelial cells. Growth of vascular smooth muscles, increase in platelet adhesion and oxidation of LDL followed by its deposition in the vascular wall and the activation of blood coagulation cascade (Baszczuk and Kopczynski 2014; McCully 2016 and McCully 2017). 
Nowadays, the use of complementary and/or alternative medicine and especially the consumption of botanicals have been increasing worldwide, because of supposedly less frequent side effects when compared to therapeutic agents (Saravanan and Pari 2008).

The wide use of genus Terminalia in traditional medicine for the treatment of various diseases may be in part due to their antioxidant potency. The potent antioxidant activity of $T$. muelleri leaf extract which might be attributed to its high tannins and flavonoids content (Nouran et al., 2017). The pharmacological activities of ellagitannins are attributed mainly to their potent free radical scavenging effect, chelation of excess metal ions, reduction of lipid peroxidation, and modulation of antioxidant defenses (Nwanna and Oboh 2007).

TM extract at the investigated dose $(200 \mathrm{mg} / \mathrm{kg})$ significantly improved the lipid profile indices, redox status of the heart, and cardiac biomarker investigated in the current study. TM extract displayed a significant decrease in total cholesterol, triglycerides and significantly increased HDL-C levels. The lowering effect of TM extract on total cholesterol and triglycerides levels could be mainly attributed to the presence of flavonoids (El-Kashak et al., 2017), that play a role in decreasing the activity of both hepatic enzyme 3-hydroxy-3-methyl glutaryl conenzyme A (HMG-CoA) reductase and acyl conzyme A: cholestrole acyl transferase (ACAT), or may be due to the presence of some HMG-CoA reductase inhibitorsas as described by Marzouk et al., (2013).

Treatment with TM extract markedly increased the cardiac content of GSH and reduced MDA level in cardiac tissue. The enhancement of these antioxidant defenses could be clearly contributed to the protective effect of TM. These findings suggest that, the protective activity of TM is attributed to its ability to enhance the antioxidant defense status that reduced the rate of lipid peroxidation and to guard against the pathological changes induced by STZ intoxication in the heart tissues. The obtained inecrease in Nrf2 after treatment with TM is the same line with the studies of Satta et al., (2017) who demonestrated that, the activation of Nrf2 by natural and synthetic substances reduces high glucoseelicited oxidative damage in cultured cardiomyocytes and prevents the development of DM in animal models of DM.

In the present study the diabetic rats treated with TM extract elicited a significant protective effect by restoring AST enzymatic activity to be near to the normal levels in association with a significant decrease in the enzymatic activity of CK-MB indicating a good cardio-protection of the tested extract. The cardio protective effect of TM was confirmed by the recorded decrease in cTn-1 indicating a preserved cardiac integrity and improved function. In addition, the reduction in the Hcy level in diabetic group treated with TM supports the efficient role Terminalia in protecting the heart against the risk factor of hypercysteinomia. This finding suggestes that several active components of $\mathrm{T} \mathrm{M}$ extract have antioxidant and antiperoxidant activities that provoke the capacity of endogenous antioxidant systems and protect against one of the most serious factors of diabetes complications.

Pioglitazone is used in the current study as reference drug. Pioglitazone is an effective oral antidiabetic agent; it is widely prescribed in the management of type- 2 diabetes mellitus (T2DM), also known as non-insulin-dependent diabetes mellitus or adult-onset diabetes (Sweetman, 2002). Pioglitazone is a potent and highly selective agonist for peroxisome proliferator activated receptor gamma (PPAR $\gamma$ ). Activation of PPAR $\gamma$ nuclear receptors modulates the transcription of a number of insulin-responsive genes involved in the control of glucose and lipid metabolism (Belcher et al., 2005). It is well established that pioglitazone enhances peripheral glucose uptake, improves insulin sensitivity in patients with T2DM (Martines et al., 2002), and prevents progression to T2DM in patients with impaired glucose tolerance and insulin resistance (Durbin, 2004). Data of this study clearly proved the potential role of TM extract in improving the glucose level and its efficacy in maintaining lipid profile in diabetic rats that may be indicated both the examined TM extract and chosen reference drug sharing the same mechanism.

\section{Conclusion}

Terminalia muelleri (TM) extract significantly ameliorates the severity of STZ-induced hyperglycemia through improving the oxidative status with protecting the heart tissue. These TM actions could be mediated by reducing lipid peroxidation process with increasing the antioxidant status of the heart and restoring the lipid profile. These data suggest that TM might be an effective 
antidiabetic agent in the prevention of diabetic complication. TM extract could help in combating against oxidation-caused heart injury.

\section{References}

Abbot, B. et al., 1984. Creatinine kinase. Kaplan A et al., Clin Chem. The C.V. Mosby Co. St Louis. Toronto. Princeton, 1112-1116.

Al-Lawati, 2017. Diabetes Mellitus: A Local and Global Public Health Emergency! Oman Med J., 32(3): 177-179.

Almeida, D.A., C.P. Braga, E.L. Novelli, and A.A. Fernandes, 2012. Evaluation of lipid profile and oxidative stress in STZ induced rats treated with antioxidant vitamin. Brazilian Archives of Biology and Technology, 55(4):527- 536.

Asmat, U., K. Abad, and K. Ismail, 2016. Diabetes mellitus and oxidative stress A concise Saudi pharmaceutical journal review, 24:547-553.

Bansal, S., S. Kapoor, G.P. Singh, and S. Ya, 2016. Serum homocysteine levels in type 2 diabetes mellitus patients. International Journal of Contemporary Medical Research, 3(11):77-83.

Baszczuk, A. and Z. Kopczynski, 2014. Hyperhomocysteinemia in patients with cardiovascular disease. Postepy Hig Med Dosw., 568-579.

Belcastro, V. and P. Striano, 2012. Antiepileptic drugs, hyperhomocysteinemia and B-vitamins supplementation in patients with epilepsy. Epilepsy Res.,102[1-2]:1-7. [DOI: 10.1016/ j.eplepsyres. 2012. 07.003].

Belcher, G., C. Lambert, G. Edwards, and R. Urquhart, 2005. Safety and tolerability of pioglitazone, metformin, and gliclazide in the treatment of type 2 diabetes. Diabetes Res Clin Pract., 70(1):5362 .

Bergmeyer, H.U., M. Horder and R. Rej, 1986. Approved recommendation (1985) on IFCC method for aspartate aminotransferase. J Clin Chem Clin Biochem., 24(7): 497-510.

Beutler, E., O. Duron, and B.M. Kelly, 1963. Improved method for determination of blood glutathione. J. Lab. and Clin. Med., 61 (5): 882-888.

Buege, J.A. and S.D. Aust, 1978. Microsomal lipid peroxidation. Methods in Enzymology, 52: 302310.

Chen, Q.M. and A.J. Maltagliati, 2018. Nrf2 at the heart of oxidative stress and cardiac protection. Physiol. Genomics, 50(2): 77-97.

Dawson, B. and R.G. Trapp, 2004. Basic and clinical biostatistics, Appleton and Lange, $4^{\text {th }}$ edition Mc Graw-Hill companies, Inc., USA. Dhingra, D.

Dean, K.J., 1998. Biochemistry and molecular biology of troponins I and T.Cardiac marker, 193-204.

Durbin, R.J., 2004. Thiazolidinedione therapy in the prevention/delay of type 2 diabetes in patients with impaired glucose tolerance and insulin resistance.Diabetes Obes Metab., 6(4):280-285.

Einarson, T.R., A. Annabel, C. Ludwig, and H. Ulrik, 2018. Prevalence of cardiovascular disease in type 2 diabetes: a systematic literature review of scientific evidence from across the world in 2007 -2017. Cardiovascular Diabetology, 17 (83): 19 pages

El-Kashak, W.A., S.M. Osman, A.H. Gaara, S.A. El-Toumy, T.K. Mohamed, and I. Brouard, 2017. Phenolic metabolites, biological activities, and isolated compounds of Terminalia muelleri extract Pharmaceutical Biology, 55(1): 2277-2284.

Fahmy, A.E., M.M. Abdel-Daim, M. Karonen, and A.N. Singab, 2016. Protective effect of Terminalia muelleri against carbon tetrachloride-induced hepato and nephro-toxicity in mice and characterization of its bioactive constituents. Pharm Biol., 54:303-313.

Fossati, P. and L. Prencipe, 1982. Serum Triglycerides Determined Colorimetrically with an Enzyme that Produces Hydrogen Peroxide. Clinical Chemistry, 28 (10): 2077-2080.

Friedewald, W.T., R.I. Levy and D.S. Fredrickson, 1972. Estimation of the concentration of lowdensity lipoprotein cholesterol in plasma without use of the preparative ultracentrifuge. Clin. Chem., 18 (6): 499-502.

Hu, X., M. Rajesh, J. Zhang, S. Zhou, S. Wang, J. Sum, Y. Tan, Y. Zeng and L. Cai, 2018. Protection by dimethyl fumarate against diabetic cardiomyopathy in type 1 diabetic mice likely via activation of nuclear factor erythroid-2 related factor 2.Toxicol Lett., 287: 131-41. 
Khan, M.R. and D. Ahmed, 2009. Protective effects of Digera muricata (L.) Mart. on testis against oxidative stress of carbon tetrachloride in rat. Food and Chemical Toxicology, 47(6):1393-1399.

Kubben, N., W. Zhang, L. Wang, T.C. Voss, J. Yang, J. Qu, G.H. Liu, and T. Misteli, 2016. Repression of the antioxidant NRF2 pathway in premature aging. Cell, 165 (6):1361-1374.

Lee, H. and M. Kang, 2013. Effect of the magnetized water supplementation on blood glucose, lymphocyte DNA damage, antioxidant status, and lipid profiles in STZ-induced rats. Nutrition Research and Practice, 7 (1): 34-42.

Mahmood, S., A. Talat, S. Karim, R. Khurshid, and A. Zia, 2011. Effect of cinnamon extract on blood glucose level and lipid profile in alloxan induced diabetes rats. Pak. J. Physiol., 7(1): 13 -16.

Martines, F.M., F.L. Visseren, J. Lemay, E.J. De Koning, and T.J. Rabelink, 2002. Metabolic and additional vascular effects of thiazolidinediones. Drugs, 62(10):1463-1480.

Marzouk, M. A.M. Soliman, and T.Y. Omar, 2013. Hypoglycemic and antioxidative effects of fenugreek and termis seeds powder in streptozotocin-diabetic rats. European Review for Medical and Pharmacological Sciences, 17(4):559- 565.

Matough, F.A., S.B. Budin, Z.A. Hamid, N. Alwahaibi, and J. Mohamed, 2012. The role of oxidative stress and antioxidants in diabetic complications.SQU Medical Journal, 12 (1): 5-18.

McCully, K.S., 2016. Homocysteine, infections, polyamines, oxidative metabolism and the pathogenesis of dementia and atherosclerosis.J Alzheimers Dis., 54(4): 1283-1290.

McCully, K.S., 2017. Hyperhomocysteinemia, suppressed immunity and altered oxidative metabolism caused by pathogenic microbes in atherosclerosis and dementia. Front Aging Neurosci., 9(324):1-12.

Modak, M., P. Dixit, J. Londhe, S. Ghaskadbi, and T.P.A. Devasagayam, 2007. Indian herbs and herbal drugs used for the treatment of diabetes. J. Clin. Biochem. Nutr., 40 (3): 163-173.

Moussa, S.A., 2008. Oxidative stress in diabetes mellitus. Romanian J. Biophys., 18 (3): 225-236.

Nouran, M., Fahmy, E. Al-Sayed and A.N. Singab, 2017. Phytochemical screening and antioxidant activity of Terminalia muelleri benth. leaf extract. Archives of Pharmaceutical Sciences Ain Shams University, 1(1):1-4

Nwaneri-Chidozie, V.O., O.E. Yakubu, J.O. Sidikat, P. Phebe, and K.C. Lele, 2014. Lipid profile status of streptozotocin-induced diabetic rats treated with ethanol, n-Hexane and aqueous extracts of Vitex doniana leaves. Research Journal of Pharmaceutical, Biological and Chemical Sciences, 5(2): 40-49.

Nwanna, E.E. and G. Oboh, 2007. Antioxidant and Hepatoprotective Properties of Polyphenol Extracts from Telfairia occidentalis (Fluted Pumpkin) Leaves on Acetaminophen Induced Liver Damage. Pakistan Journal of Biological Sciences, 10(16): 2682-2687.

Paget, G.E. and J.M. Barnes, 1964. In: "toxicity tests" volume (1) chapter (6) pp 135. Editor Laurance, D.R. and Bacharach A.L. Academic press, London, New York.

Parkash, J., D.N. Prasad, M. Shahnaz and D. Dev, 2018. Herbs as traditional medicines: A review. Journal of drug delivery and therapeutics, 8 (5):146-150.

Pitocco, D., M. Tesauro, R. Alessandro, G. Ghirlanda, and C. Cardillo., 2013. Oxidative Stress in Diabetes: Implications for Vascular and Other Complications. I J. Mol. Sci., 14: 21525-21550.

Poznyak, A., A.V. Grechko, P. Poggio, V.A. Myasoedova, V. Alfieri, and A.N. Orekhov, 2020. The Diabetes Mellitus-Atherosclerosis Connection: The Role of Lipid and Glucose Metabolism and Chronic Inflammation. International Journal of Molecular Sciences, 21(1835):1-13.

Rajeswari G, and V. Rajagopalan, 2013. Evaluation of anti-diabetic effects of Chrysopogon zizanioides Linn root extracts in streptozotocin induced diabetic Wistar rats. Journal of Scientific and Innovative Research, 2(3):555-574.

Richmond, W., 1973. Preparation and properties of a cholesterol oxidase nocardia species and its application to the enzymatic assay of total cholesterol in serum. Clin. Chem., 19(12):1350-1356.

Said, M.M., H.H. Abd El-Latif, A.M. Nour, M.S. Zohni, and A.A. Abd El-Rahman, 2000. Biochemical effects of some natural products on normal rats and their protective effects against hyperglycemia. J. Drug Res. Egypt, 23: 239-250.

Saravanan, G. and L. Pari, 2008. Hypoglycemic and antihyperglycemic effect of Syzygium cuminh bark in streptozotocin-induced diabetic rats. Journal of Pharmacology and Toxicology, 3(1): 110 . 
Satta, S., A.M. Mahmoud, F.L. Wilkinson, M.Y. Alexander, and S.J. White, 2017. The Role of Nrf2 in Cardiovascular Function and Disease. Hindawi Oxidative Medicine and Cellular Longevity Volume 2017, Article ID 9237263, 18 pages. https://doi.org/10.1155/2017/9237263.

Shaikh, M.K., B.R. Devrajani, A. Shaikh, S.A. Shah, S. Shaikh, and D. Singh, 2012. Plasma homocysteine level in patients with diabetes mellitus World Applied Sciences Journal, 16 (9): 1269-1273.

Shipchandler, M.T. and E.G. Moore, 1995. Rapid, fully automated measurement of plasma homocyst (e) ine with the Abbott IMx analyzer.Clin Chem., 41 (7): 991-994.

Subash, B., P., P. Prabuseenivasan, and S. Ignacimuthu, 2007. Cinnamaldehyde a potential antidiabetic agent. Phytomedicine, 14(1):15-22.

Sweetman, C.S., 2002. Martindale the complete drug reference $33^{\text {th }}$ ed. London: Pharmaceutical Press:

Trinder, P., 1969. Enzymatic method of glucose estimation. Ann Clin Biochem., 6: 24-33.

Warnick, G.R. and J.J. Albers, 1978. Heparin- $\mathrm{Mn}^{2+}$ quantitation of high density lipoprotein cholesterol: an ultrafiltration procedure for lipemic samples. Clin. Chem., 24: 900-904.

Wang, Y.B., Z.M. Ge, W.Q. Kang, Z.X. Lian, J. Yao, and C.Y. Zhou, 2015. Rutin alleviates diabetic cardiomyopathy in a rat model of type 2 diabetes. Experimental and Therapeutic Medicine, 9: 451-455.

Wang, G., X. Song, L. Zhao, Z. Li, and B. Liu, 2018. Resveratrol prevents diabetic cardiomyopathy by increasing Nrf2 expression and transcriptional activity. Biomed Res Int., volume 2018 ID 2150218, 13 page

Wu, F., B. Yu, X. Zhang, and Y. Zhang, 2017. Cardioprotective effect of Notch signaling on the development of myocardial infarction complicated by diabetes mellitus. Experimental and therapeutic medicine, 14(4): 3447-3454.

Zang, H., R.O. Mathew, and T. Cui, 2020. The Dark Side of Nrf2 in the Heart. Frontiers in physiology, 11(722):1-10. 